\title{
Efeito do treinamento com medicine balls na velocidade da bola do polo aquático
}

http://dx.doi.org/10.11606/1807-5509201900030487

\author{
Querim Goulart ZANETTE* $^{*}$ \\ Guilherme TUCHER ** \\ Marcos FRANKEN* \\ Camila Dias de CASTRO* \\ Flávio Antônio de Souza CASTRO*
}

*Universidade Federal do Rio Grande do Sul, Porto Alegre, RS, Brasil.

${ }^{* *}$ Instituto Superior de Educação do Município de Itaperuna, Itaperuna, RJ, Brasil.

\section{Resumo}

0 objetivo deste estudo foi verificar o efeito do treinamento da força muscular na velocidade da bola em arremesso a gol realizado por jogadores de polo aquático. Participaram 23 jogadores de polo aquático do sexo masculino divididos aleatoriamente em grupos experimental $(G E, n=13)$ e controle $(G C, n=10)$. Foi realizada a mensuração da velocidade da bola nos arremessos de pênalti com um radar pré- e pós-treinamento de seis semanas. GE e GC executaram o mesmo treinamento de polo aquático na água, cabendo somente ao GE o treinamento da força muscular fora da água por meio da simulação de arremessos com medicine balls de 1,0 e $1,5 \mathrm{~kg}$. Os resultados indicaram incremento significativo $(p<0,05)$ na velocidade da bola após arremesso de pênalti apenas no GE $\left(15,6 \pm 1,8 \mathrm{~m} \cdot \mathrm{s}^{-1}\right.$ para 16,3 $\left.\pm 1,5 \mathrm{~m} \cdot \mathrm{s}^{-1}\right) .0$ treinamento da força muscular por meio do arremesso fora da água com medicine balls mais pesadas que a bola utilizada no jogo de polo aquático mostrou-se capaz de incrementar a sua velocidade após arremesso a gol realizado na distância do pênalti.

Palavras-chave: Adaptação ao Treinamento; Treinamento de Força Muscular; Desempenho Esportivo; Carga de Treinamento.

\section{Introdução}

O polo aquático (PAq) é uma modalidade intermitente de alta intensidade, com predomínio de deslocamentos rápidos em diversos tipos de nado. Passes, arremessos e disputas pela posse da bola são frequentes, de grande importância e envolvem aproximadamente $36 \%$ do tempo total de cada jogo; sendo os outros $34 \%$ do tempo atividades de natação ${ }^{1,2}$. O desempenho competitivo dos atletas na modalidade depende de características antropométricas e do desenvolvimento de capacidades condicionantes e coordenativas ${ }^{3-5}$, como resistência, agilidade, velocidade e potência, tanto de membros inferiores (relacionada à alçada executada com movimento de pernas em eggbeater para subir na água), quanto de membros superiores (relacionada aos arremessos a gol e aos bloqueios dos arremessos) $)^{4,5}$.

Arremessar é uma habilidade técnica básica em um jogo de $\mathrm{PAq}^{6}$. Assim, um dos principais objetivos do treinamento na modalidade é a efetividade no arremesso, que está relacionada a fatores como velocidade e precisão ${ }^{7,8}$. No arremesso por sobre a cabeça (overhead) a bola vem por trás do corpo, passa por cima da cabeça e o movimento é finalizado à frente do corpo. Seu objetivo é fazer a bola alcançar alta velocidade ${ }^{8}$ no arremesso direcionado à baliza do adversário. Este tipo de arremesso é o movimento mais utilizado (em torno de $90 \%$ ) para passes e arremessos a gol por ser o mais efetivo ${ }^{4-6}$.

Embora o treino físico-técnico-tático regularmente realizado na água possa fazer com que os atletas melhorem seus arremessos no $\mathrm{PAq}^{5}$, a realização do treinamento da força muscular também demonstrou ser uma estratégia útil para o incremento da velocidade do arrremesso ${ }^{5}$. VeLIZ et al. ${ }^{5}$ avaliaram a velocidade da bola pós-arremesso 
em jogadores de PAq de nível nacional. Todos os jogadores realizaram o treinamento na água, entretanto o grupo experimental também realizou o treinamento tradicional da força muscular (e.g., supino, desenvolvimento, barra fixa). Ao final do período de 18 semanas de treinamento foi encontrado incremento significativo $(2,76 \%)$ na velocidade da bola somente para o grupo que realizou o treinamento da força muscular. Já SKOUFAS et al. ${ }^{9}$ avaliaram a velocidade do arremesso da bola no handball, após treinamento com bolas mais leves que as do jogo. Os autores também encontraram incremento na velocidade da bola após o treinamento (entre $4,3 \%$ e $6,6 \%$ ).

Pelo exposto, percebe-se que o treinamento adicional ao técnico-tático, bem como a modulação do peso da bola pode induzir resultados positivos na velocidade do arremesso ${ }^{4-11}$. Entretanto, destaca-se que o treinamento empregando o gesto do arremesso com implementos mais leves ou pesados que a bola utilizada em jogo apresenta-se como uma estratégia de maior especificidade e pouco dispendiosa, fácil e prática de ser mantida no programa de treinamento de jogadores de PAq. Assim, considerando a importância da velocidade atingida pela bola para a efetividade do arremesso no $\mathrm{PAq}^{7,8}$, a objetivo deste estudo foi verificar o efeito do treinamento com medicine balls na velocidade da bola em arremesso a gol realizado por jogadores de polo aquático. A hipótese estabelecida é de que o treinamento com medicine balls realizado fora da água incrementa a velocidade da bola no arremesso realizado dentro da água.

\section{Método}

\section{Amostra}

Participaram deste estudo 23 jogadores de PAq do sexo masculino que foram divididos aleatoriamente em grupo experimental (GE, n =13) e grupo controle $(\mathrm{GC}, \mathrm{n}=10)$. Ambos os grupos realizaram o treinamento de rotina da modalidade dentro da água, que consistia, geralmente, de aquecimento em nados variados, séries de velocidade de nado, séries de resistência aeróbica de natação, séries de perna em egg-beater, troca de passes, arremessos e treinamento tático. Todos os participantes treinavam três vezes por

semana, por 90 min em cada sessão e participavam de competiçôes estaduais e regionais, com quatro competiçôes por ano, em média 4 jogos a cada dois meses. Entretanto, somente o GE realizou o treinamento da força muscular por meio dos arremessos com medicine balls. Todos os atletas eram vinculados à Confederação Brasileira de Desportos Aquáticos (CBDA) e atuavam em uma mesma equipe, apresentando, no mínimo, dois anos de experiência competitiva. Houve perda amostral (três participantes) no GC por doença e/ou lesão que impediu a segunda avaliação. As características dos participantes são apresentadas na TABELA 1.

TABELA 1-Média e desvio-padrão de idade, estatura, massa corporal e experiência na modalidade por grupo; $\mathrm{GE}$ - grupo experimental; GC - grupo controle.

\begin{tabular}{ccccc}
\hline Grupos & Idade (anos) & Estatura (cm) & Massa (kg) & Experiência (anos) \\
\hline GE, $n=13$ & $32,2 \pm 5,2$ & $181,2 \pm 4,8$ & $85,4 \pm 9,4$ & $11,4 \pm 5,4$ \\
GC, $n=10$ & $34,7 \pm 7,0$ & $180,3 \pm 5,0$ & $85,0 \pm 11,5$ & $12,6 \pm 4,0$ \\
\hline
\end{tabular}

Antes das avaliaçóes, os participantes foram informados de todos os procedimentos metodológicos e assinaram o termo de consentimento livre e esclarecido concordando com a participação no estudo. $\mathrm{O}$ estudo, realizado de acordo com a Declaração de Helsinque para a pesquisa em seres humanos, foi aprovado pelo Comitê de Ética em Pesquisa da Instituição onde foi realizado, sob parecer número 20659.

No primeiro dia de avaliação foram explicados todos os procedimentos e fornecido o Termo de Consentimento Livre e Esclarecido aos atletas, que foi lido e assinado por todos. Logo após, foram obtidos os dados de idade, estatura e massa corporal, bem como o tempo de experiência na modalidade, em anos.

\section{Avaliação da velocidade da bola}

A velocidade da bola após arremesso da posição 
de pênalti ( 5 m de distância da baliza) (FIGURA 1a) foi obtida em dois momentos: pré e pós-treinamento de seis semanas de duração. A velocidade da bola foi mensurada com uma pistola radar (Bushnell, EUA), conforme descrito anteriormente por SKOUFAS et al. ${ }^{9}$. Esse radar contém um transmissor de radiofrequência (FIGURA 1b) que opera na banda $\mathrm{K}$, uma micro-onda eletromagnética que cobre frequências de 26,5 a $40 \mathrm{gHz}$, utilizada em satélites de comunicação e radares para detectar a velocidade de objetos em movimento. $\mathrm{O}$ radar foi posicionado atrás da baliza (FIGURA 1b).
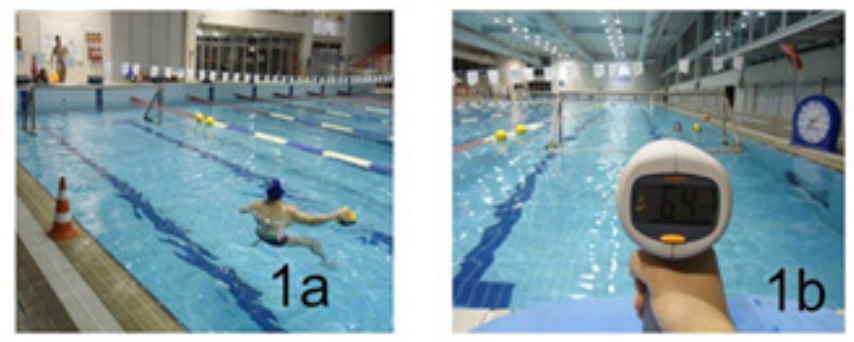

FIGURA 1 -Posicionamento do jogador para executar o arremesso cuja velocidade da bola foi avaliada (1a); radar a uma distância de cinco metros atrás da baliza (1b).

Cada participante arremessou cinco vezes, sempre por sobre a cabeça, pré e pós-treinamento, em posição perfeitamente perpendicular à baliza de tamanho oficial. Os atletas foram instruídos a arremessar no meio do gol, sem a bola quicar na água. A posição perpendicular foi controlada, a fim de que possíveis alterações na medida da velocidade da bola não alterassem o resultado final. Foi adotado o intervalo de um minuto entre cada tentativa a fim de se evitar possíveis efeitos da fadiga. Apenas arremessos que foram diretamente para a rede, sem tocar nas balizas e na água foram validados. Dos três valores intermediários, obtidos a partir das cinco tentativas, foi calculada a média de velocidade da bola para cada indivíduo. Bolas oficiais masculinas de PAq (marca Penalty, WP3, massa de 0,450 kg) foram utilizadas e não havia goleiro posicionado para a defesa dos arremessos. Foram calculados os erros técnicos de medida, absoluto e relativo, de acordo com proposto por Perini et al. ${ }^{12}$.

Previamente às medidas da velocidade da bola, foi realizado aquecimento padrão na piscina, que consistia de $300 \mathrm{~m}$ de natação livre e três minutos de troca de passes em duplas. Durante o período de mensuração da velocidade da bola (pré e pós treinamento) e nas seis semanas de duração do treino da força muscular por meio de arremesso com medicine balls a rotina de treinos de PAq e demais atividades realizadas pelos jogadores não foram alteradas.

Treinamento de arremesso com medicine balls fora da água

O programa de treinamento da força muscular com medicine balls foi realizado sempre antes da sessão de treino na água, teve duração de seis semanas e frequência de duas sessões semanais. Foram realizados arremessos por sobre a cabeça utilizando medicine balls contra um alvo fixado a uma distância de $4 \mathrm{~m}$ a frente do atleta. Os atletas foram instruídos a realizar os arremessos em máximas velocidades. Nas quatro primeiras semanas foi utilizada uma medicine ball de $1,0 \mathrm{~kg}$ e $195 \mathrm{~mm}$ de diâmetro. Nas duas últimas semanas, uma medicine ball de $1,5 \mathrm{~kg}$ e $205 \mathrm{~mm}$ de diâmetro. O emprego dessas cargas foi relacionada ao incremento de, aproximadamente, 100 a $200 \%$ da massa da bola de PAq normalmente utilizada.

$\mathrm{Na}$ primeira semana do programa de treinamento da força muscular foi realizado um total de 20 arremessos (duas séries de 10 repetições) para cada sessão de treino. $\mathrm{O}$ intervalo entre as repetiçōes era mínimo, apenas o tempo de recuperar a bola e repetir o gesto. Em todas as sessôes houve um intervalo de repouso passivo de um minuto entre o aquecimento e as séries, e entre as séries ${ }^{13}$. Houve incremento de 10 repetiçóes na quantidade de arremessos entre a primeira e a quarta semana (alcançando cinco séries de dez repetiçôes na quarta semana). Nas duas últimas semanas houve incremento de carga $(1,5 \mathrm{~kg})$ e manutenção no número de repetições (5 séries de 10 repetições). Sempre antes de cada sessão de treino, foi realizado aquecimento padrão, que consistia de 10 arremessos leves, na distância de $4 \mathrm{~m}$ do alvo, com as medicine balls que seriam utilizadas na sessão de treino. 
O treinamento da força muscular com medicine balls foi realizado no horário e local de treino dos atletas, mas fora da piscina, ficando o indivíduo posicionado com o pé contrário ao braço de arremesso à frente em contato com o solo e o joelho contralateral flexionado e apoiado no solo. Durante a realização dos arremessos com medicine balls foi sempre enfatizada a execução correta do gesto, movimento controlado pelo avaliador de forma qualitativa com os seguintes parâmetros: (1) início do movimento atrás da cabeça; (2) realização de rotação do tronco ao longo do movimento; (3) finalização da ação à frente do rosto do executante, término com a rotação do tronco para o lado oposto do braço de arremesso e extensão do cotovelo (FIGURA 2). O avaliador fornecia feedback ao atleta sobre a qualidade dos arremessos de acordo com os parâmetros citados.

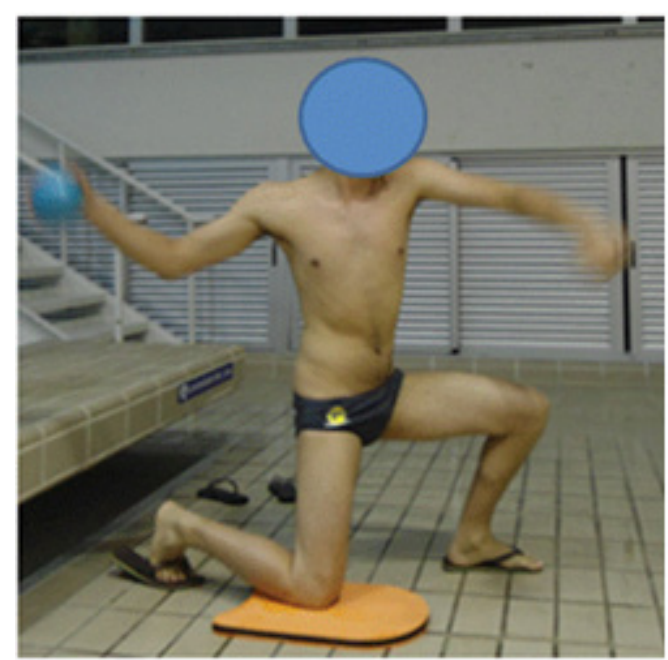

FIGURA 2 -Posicionamento do atleta para o treinamento de força de arremesso com medicine balls fora da água.

\section{Análise estatística}

Foi verificada a normalidade de todas as variáveis obtidas (antropométricas e velocidade da bola, pré e pós-treinamento) com o Teste de Shapiro-Wilk e, após, calculadas médias e desviospadrão. A variação percentual da velocidade da bola $(\Delta \%)$, pré e pós, foi calculada assumindo-se o valor pré como $100 \%$. As características dos participantes e o $\Delta \%$ foram comparadas, entre os grupos, com a aplicação de um Teste t de Student para dados independentes. Já, para a comparação dos resultados obtidos nos dois momentos de coleta (velocidade da bola) e entre os dois grupos (GE e GC) foi aplicada uma ANOVA de modelo misto $(2 \times 2)$, sendo fatores os grupos e os momentos pré e pós-treinamento. Tamanho de efeito do fator grupo sobre a velocidade da bola, em cada momento de avaliação, e sobre o $\Delta \%$ foi verificado com aplicação do teste d de Cohen, cujos critérios de interpretação foram: de 0 a 0,2 : trivial; de 0,21 a 0,6 : pequeno; de 0,61 a 1,2: moderado; de 1,21 a 2,0: grande e maior que 2,0: muito grande ${ }^{14}$. Todos os dados foram processados no software SPSS 17.0. O nível de significância adotado foi de 5\%.

\section{Resultados}

Não foram encontradas diferenças entre os grupos experimental e controle para idade, estatura, massa e experiência. Os valores de velocidade da bola para todos os participantes (geral), por grupos e por momentos (pré e pós-treinamento), bem como os valores de $\Delta \%$ estão apresentados na TABELA 2. Sobre a velocidade da bola houve interação significativa grupo $\mathrm{x}$ momento, com valores semelhantes entre os grupos no momento pré e maiores valores para o GE no momento pós, tanto em relação ao GC no momento pós, quanto a seu próprio momento pré-treinamento. A variação de incremento na velocidade, em média, foi maior no GE do que no GC, este com variação negativa e maior variabilidade. 
TABELA 2 -Média e desvio-padrão de velocidade da bola (Vel) nos momentos pré e pós-treinamento por grupos (GE: grupo experimental e GC: grupo controle).

\begin{tabular}{|c|c|c|c|}
\hline Participantes & Vel pré $\left(\mathrm{m} . \mathrm{s}^{-1}\right)$ & Vel pós $\left(\mathrm{m} \cdot \mathrm{s}^{-1}\right)$ & $\Delta \%$ \\
\hline GE $n=13$ & $15,6 \pm 1,8^{\mathrm{a}}$ & $16,3 \pm 1,5^{\mathrm{a}}$ & $5,0 \pm 4,5^{*}$ \\
\hline GC, $n=10$ & $15,8 \pm 1,2^{\mathrm{a}}$ & $15,2 \pm 1,5^{\mathrm{a}}$ & $-3,7 \pm 11,4^{*}$ \\
\hline
\end{tabular}

O tamanho do efeito dos grupos sobre a velocidade da bola, no momento pré, foi de 0,12 (trivial), no momento pós, de 1,8 (grande) e sobre o $\Delta \%$, foi de 1,0 (moderado). Os erros técnicos de medida da velocidade, absoluto e relativo, foram de, respectivamente, $0,44 \mathrm{~m} \cdot \mathrm{s}^{-1}$ e $2,84 \%$. As mudanças encontradas para velocidade da bola no GE foram acima do erro mensurado.

\section{Discussão}

A velocidade com que a bola é arremessada ao gol é um dos fatores determinantes para o sucesso no PAq, já que altas velocidades de arremesso dificultam a ação dos defensores, especialmente do goleiro ${ }^{11}$. Assim, o presente estudo teve como objetivo verificar o efeito do treinamento da força muscular com medicine balls fora da água na velocidade da bola após arremesso dentro da água. Com este propósito foi realizado por seis semanas um programa de treinamento da força muscular que simulou o gesto do arremesso do PAq fora da água. Este treinamento foi capaz de incrementar a velocidade da bola após arremesso dentro da água.

ABRALDES et al. ${ }^{6}$ encontraram velocidades médias após arremessos de pênalti de 15,9 $\pm 1,4 \mathrm{~m} . \mathrm{s}^{-1}$ para jogadoras e de 20,2 \pm 1,4 m.s-1 para jogadores de nível internacional de PAq. De forma semelhante, MCCLUSKEY et al. ${ }^{11}$ encontraram velocidades médias

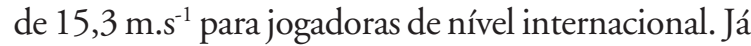
VeLIZ et al. ${ }^{5}$, ao avaliarem os efeitos do treinamento tradicional de força muscular sobre a velocidade da bola, encontraram, em jogadores de nível nacional no pós-treinamento valor de $18,2 \pm 2,1 \mathrm{~m} \cdot \mathrm{s}^{-1}$. Percebe-se que os valores de velocidade da bola registrados no presente estudo (variando de $15,6 \pm 1,8$ a $16,3 \pm 1,5$ m. $s^{-1}$ - TABELA 2), realizado apenas com jogadores do sexo masculino de nível regional, estão mais próximos aos valores de jogadoras de nível internacional do que de jogadores. Tal resultado pode ser explicado pelo nível dos jogadores participantes, bem como pelas horas semanais dedicadas ao treinamento.

$\mathrm{O}$ treinamento proposto neste estudo utilizou bolas com massas de 1 e $1,5 \mathrm{~kg}$, em contraste com a bola de PAq, cujo modelo masculino possui, em média, 0,450 kg. Não foram encontrados estudos similares no PAq que permitissem uma melhor comparação. De qualquer forma, os resultados do presente estudo corroboram com os obtidos por Skoufas et al. ${ }^{9}$ e DeChechi et al. ${ }^{15}$. Estes estudos (ambos com jogadores de handebol) utilizaram, respectivamente, bolas mais leves e mais pesadas, durante o treinamento de força.

SKOUFAS et al. ${ }^{9}$ treinaram dois grupos de jogadores de handebol ao longo de 20 semanas. Um grupo utilizou bolas $20 \%$ mais leves que as habituais e o outro treinou com bolas de handebol sem alteração de massa. Os dois grupos realizaram programa de treinamento que consistiu de 9-16 séries de 6 arremessos máximos durante cada sessão de treinamento. Durante as dez primeiras semanas o treinamento foi voltado para aprender, melhorar e padronizar todos os fundamentos técnicos do handebol. Após este período os indivíduos foram treinados por 10 semanas adicionais. Neste último momento foram utilizadas as bolas com pesos diferentes. A questão de melhora da técnica também foi enfatizada por Skoufas et al. ${ }^{9}$. Dificilmente podem-se separar os efeitos do treinamento específico entre aqueles decorrentes da força/potencia e da técnica. Ambos os estudos (o presente e o de SKOUFAS et al..$\left.^{9}\right)$ podem ter obtido aumento na velocidade da bola por (1) incremento de força/potência, por (2) melhoria da técnica de execução do gesto, ou, ainda, por (3) diferentes contribuições de força, potência e técnica. Como o treinamento da força muscular com medicine balls realizado no presente estudo teve execução gestual qualitativamente controlada em relação à técnica, a velocidade da bola após o arremesso pode ter sido incrementada também por sua melhoria técnica. Indício de tal resultado foi o $\Delta \%$ do GE em comparação ao GC, que não realizou o treinamento proposto (TABELA 2).
Legenda: ${ }^{a}$ : interação significativa grupo x momento sobre a velocidade da bola $\left(\mathrm{F}_{1,21}=\right.$ 6,41; $\mathrm{p}=0,019)$; *: comparação entre os grupos do $\Delta \%\left(\mathrm{t}_{21}=-2,53 ; \mathrm{p}\right.$ $=0,019)$. 
Já Dechechi et al. ${ }^{15}$, em estudo também com jogadores de handebol, utilizaram treinamento de arremesso com medicine balls cujas massas variaram entre 1 e $3 \mathrm{~kg}$ (bola de handebol masculina possui massa de $0,425 \mathrm{~kg}$ a $0,475 \mathrm{~kg}$ ), ao longo de diversos ciclos de treinamento. Porém, diferente do presente estudo, que utilizou o próprio arremesso para se avaliar o efeito decorrente do treinamento realizado, DeCHeCHI et al. ${ }^{15}$ utilizaram o teste de arremesso de medicine ball para treinar e avaliar a potência de membros superiores. Mesmo assim, houve incremento de potência relacionado à utilização dos arremessos com bola mais pesada que a utilizada na modalidade.

Maior velocidade da bola após arremesso nas diferentes modalidades esportivas depende de incremento de potência muscular, produto entre a força muscular e a velocidade de execução do gesto $^{16}$. Assim, existem diferentes possibilidades de treinamento quanto à utilização de implementos mais pesados ou mais leves do que aqueles habitualmente utilizados no esporte (como no handebol e no beisebol) para aumentar a velocidade da bola após arremesso $^{17}$. Por um lado, podem-se usar bolas cuja massa é maior do que aquelas usualmente utilizadas na modalidade específica ${ }^{15,18,19}$. Este treinamento parece focar no aumento da força muscular. Por outro lado, a utilização de bolas com massa abaixo das normalmente utilizadas na prática da modalidade ${ }^{9,18,19}$ tendem a focar o treinamento no incremento da velocidade gestual. Ambas as possibilidades de treino parecem incrementar a potência do gesto, um pelo incremento da força, outro pelo incremento da velocidade. Optou-se, no presente estudo, pela primeira condição de treinamento.

VeLIZ et al. ${ }^{5}$ investigaram os efeitos do treinamento de força sobre habilidades específicas no PAq (velocidade do arremesso, velocidade de natação e altura de salto) em jogadores de elite. Neste estudo de Veliz et al. ${ }^{5}$, o treinamento de força foi realizado fora da água, com exercícios que focaram movimentos articulares gerais, como apoios de frente sobre o solo, agachamentos, saltos e abdominais. Dentre outros resultados, os autores encontraram que o grupo experimental (que além do treino de PAq realizou o treino de força) incrementou de modo significativo a velocidade da bola pós-arremesso (de $17,7 \pm 1,8$ para $\left.18,2 \pm 2,1 \mathrm{~m} \cdot \mathrm{s}^{-1}\right)$. Este incremento, de aproximadamente de $2,7 \%$, foi menor do que o incremento obtido no presente estudo $(5,0 \pm 4,5 \%)$. Porém, além do treinamento utilizado por VeLIZ et al. ${ }^{5}$ não ter focado no gesto do arremessar, apenas na força/potência geral, foi realizado com atletas de nível nacional, cuja possibilidade de adaptação ao treinamento é menor do que atletas de menor nível, como os do presente estudo. Ao se comparar com os resultados do presente estudo, talvez os jogadores se beneficiassem mais com ambos os tipos de treinamentos de força executados de modo periodizado $^{20}$.

Apesar da importância do treinamento de força para o arremesso nas modalidades cujo desempenho depende da força/potência muscular no arremesso, poucos estudos apresentam dados empíricos sobre os efeitos dos métodos de treinamento de força na velocidade da bola após arremesso ${ }^{5-11}$. Porém, nos últimos anos, novos estudos sobre arremesso, especialmente no PAq, vêm sendo realizados ${ }^{5,6-8,10,11}$ o que parece mostrar um aumento no interesse nessa área. Além disso, considerando a realização dos Jogos Olímpicos no Brasil, em 2016, trabalhos têm sido realizados sobre o polo aquático brasileiro ${ }^{3,21,22}$, indicando que maior atenção tem sido dispensada a esta modalidade.

Este estudo demonstrou que o treinamento de arremesso com medicine balls é capaz de incrementar a velocidade da bola no arremesso a gol. Por outro lado, pode-se considerar como um fator limitante as avaliações terem sido realizadas em ambiente controlado, fora do contexto do jogo. Durante uma partida de polo aquático há situaçôes inesperadas ${ }^{23,24}$ e o jogador arremessa sob pressão por parte do adversário, de diferentes locais e distâncias do gol3${ }^{25}$. Deste modo, sugere-se a realização de estudos semelhantes que levem em consideração a avaliação da velocidade da bola pós-arremesso durante os próprios jogos - considerando as situações específicas de desempenho. Entretanto, com a metodologia empregada há uma maior facilidade de se avaliar a velocidade da bola por não haver interferência dos demais jogadores e do goleiro.

A nossa principal conclusão é que o treinamento para o arremesso executado fora da água utilizando implementos com maior massa do que aqueles utilizados comumente no PAq (bola) é estímulo eficaz para o incremento da velocidade da bola após arremesso a gol em jogadores de nível regional. Confirmou-se a hipótese de que o treinamento da força muscular com medicine balls realizado fora da água incrementa a velocidade da bola no arremesso realizado dentro da água. Este incremento na velocidade da bola pode estar associado tanto ao aumento da potência muscular, quanto à melhoria da técnica do arremesso. 


\title{
Agradecimentos
}

Aos atletas que participaram deste estudo e a todos que se disponibilizaram para auxiliar na realizaçáo do mesmo.

\begin{abstract}
Effect of training with medicine balls on water polo ball speed

The purpose of this study was to verify the effect training in the ball speed after throw to the goal in water polo players. Twenty-three male water polo players randomly divided into experimental $(G E, n=13)$ and control $(G C, n=10)$ groups participated. The ball speed was measured in the penalty shoot with a radar, pre-and post-six weeks of training. GE and GC performed the same water-polo training in the water, but only GE performed the out-of-water resistance training by simulating pitches with 1.0 and $1.5 \mathrm{~kg}$ medicine balls. The results indicated a significant increase $(p<0.05)$ in ball velocity after penalty throw only in the GE $\left(15.6 \pm 1.8\right.$ to $\left.16.3 \pm 1.5 \mathrm{~m} . \mathrm{s}^{-1}\right)$. Resistance training by throwing off water with medicine balls heavier than the ball used in the water polo game can be able to increase its velocity after a goal throw in the distance from the penalty.
\end{abstract}

KEYwoRDs: Adaptation to Training; Resistance Training; Sport Performance; Training Load.

\section{Referências}

1. Smith HK. Applied physiology of water polo. Sports Med. 1998;26:317-334.

2. D'Auria S, Gabbett T. A time-motion analysis of international Women's water polo match play. Int J Sports Physiol Perform. 2008;3:305-319.

3. Tucher G, Castro FAS, Silva SDMQ, Garrido N, Cabral RG, Silva AJ. Relationship between origin of shot and occurrence of goals in competitive men's water polo matches. Rev Bras Cineantropom Desempenho Hum. 2014;16:136-143.

4. Ferragut C, Alcaraz PE, Vila H, Abraldes JA, Rodriguez N. Evaluation of the validity of radar for measuring throwing velocities in water polo. XIth International Symposium for Biomechanics and Medicine in Swimming; 16-19 jun. 2010; Oslo. Oslo: Biomechanics and Medicine Swimming XI; 2010. p. 77-78.

5. Veliz R, Requena B, Suarez-Arrones L, Newton RU, Villarreal ES. Effects of 18 week in-season heavy resistance and power training on throwing velocity, strength, jumping, and maximal sprint swim performance of elite male water polo players. J Streng Condit Res. 2014;28(4):1007-1014.

6. Abraldes JA, Ferragut A, Rodríguez N, Alcaraz PE, Vila H. Throwing velocity in elite water polo from different areas of the swimming pool. Port J Sport Sci. 2011;11(2):41-44.

7. Royal KA, Farrow D, Mujika I, Halson SL, Pyne D, Abernethy B. The effects of fatigue on decision making and shooting skill performance in water polo players. J Sports Sci. 2006;24(8):807-815.

8. Platanou T, Botonis P. Throwing accuracy of water polo players of different training age and fitness levels in a static position and after previous swimming. XIth International Symposium for Biomechanics and Medicine in Swimming; 16-19 jun. 2010; Oslo. Oslo: Biomechanics and Medicine Swimming XI; 2010. p. 281-283.

9. Skoufas D, Stefanidis P, Michailidis C, Kotzamanidou M. The effect of handeball training with underweighted balls on the throwing velocity of novice handball players. J Hum Mov Studies. 2003;44(4):157-171.

10. Vila Suárez H, Ferragut Fiol C, Argudo Iturriaga FM, Abraldes Valeiras JA, Rodríguez Suárez N, Alacid Cárceles F. Relación entre parâmetros antropométricos y la velocidad de lanzamiento en jugadores de waterpolo. J Hum Sport Exerc. 2009;4(1):62-74.

11. McCluskey L, Lynskey S, Leung CK, Woodhouse D, Briffa K, Hopper D. Throwing velocity and jump height in female water polo players: performance predictors. J Sci Med Sport. 2010;13:236-240.

12. Perini TA, Oliveira GL, Ornellas JS, Oliveira FP. Cálculo do erro técnico de medição em antropometria. Rev Bras Med 
Esp. 2005;11(1):81-85.

13. Fleck S, Kreamer WJ. Fundamentos do treinamento de força muscular. 2a ed. Porto Alegre: Artmed; 1999.

14. Hopkins WG. Measures of reliability in sports medicine and science. Sports Med. 2000;30(1):1-15.

15. Dechechi CJ, Machado EFA, Ide BN, Lopes CR, Brenzikofer R, Macedo DV. Estudo dos efeitos de temporada de treinamento físico sobre a performance de uma equipe de handebol feminino sub-21. Rev Bras Med Esp. 2010;16(4):295300.

16. Komi PV. Força e potência no esporte. 2a ed. Porto Alegre: Artmed; 2006.

17. Van Den Tillaar R. Effect of different training programs on the velocity of overarm throwing: a brief review. J Streng Condit Res. 2004;18(2):388-396.

18. Edwards Van Muijen AJ, Jo“ris HJJ, Kemper HCG, Van Ingen Schenau GJ. Throwing practice with different ball weights: effects on throwing velocity and muscle strength in female handball players. Sports Train Med Rehabilit. 1991;2:103-113.

19. Derenne C, Buxton BP, Hetzel RK. Effects of under- and overweighted implement training on pitching velocity. J Streng Condit Res. 1994;8(4):247-250.

20. Mcguigan M, Wright G, Fleck S. Strength training for athletes: does it really help sports performance? Int J Sports Physiol Perform. 2012;7(1):2-5.

21. Tucher G, Castro FAS, Garrido N, Da Silva AJRM. The reliability of a functional agility test for water polo. J Hum Kinetics. 2014b;41:181-190.

22. Tucher G, Canossa S, Cabral RG, Garrido ND, Castro FAS. Relação entre a eficácia da superioridade numérica temporal e o resultado da partida no polo aquático. Rev Educ Fís. 2015;26(4):541-547.

23. Falk B, Lidor R, Lander Y, Lang B. Talent identification and early development of elite water-polo players: a 2-year follow-up study. J Sports Sci. 2004;22(4):347-355.

24. Uljevic O, Esco MR, Sekulic D. Reliability, validity, and applicability of isolated and combined sport-specific tests of conditioning capacities in top-level junior water polo athletes. J Streng Condit Res. 2014;28(6):1595-1605.

25. Lupo C, Tessitore A, Minganti C, Capranica L. Notational analysis of elite and sub-elite water polo matches. J Streng Condit Res. 2010;24(1):223-229.

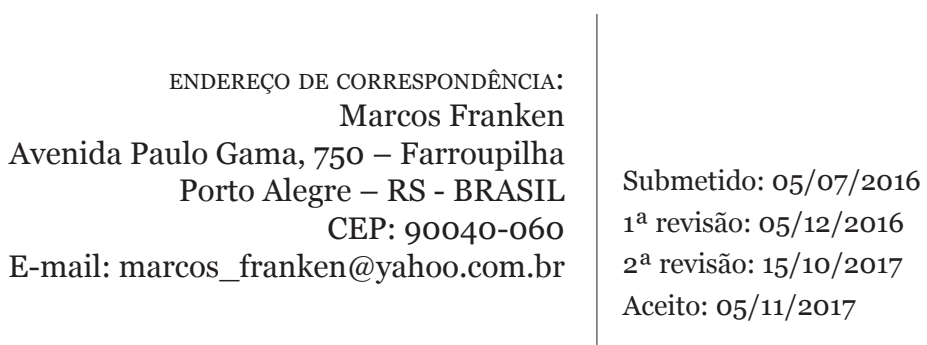

